\title{
Time and frequency transfer with the ESA/CNES ACES-PHARAO mission
}

\author{
P. Delva $\dagger$, C. Le Poncin-Lafitte, P. Laurent, F. Meynadier and P. Wolf \\ LNE/Syrte - Observatoire de Paris,CNRS,UPMC Univ Paris 06,UMR8630, 75005 Paris, France
}

\begin{abstract}
We have written a theoretical description of one-way and two-way satellite time and frequency transfer and developed a model of the micro-wave link in the frame of the ACES/PHARAO mission. This is used to write a data analysis software and a simulation to test it. A very short description of the mission and of the micro-wave link is given here. A detailed description can be found in Delva et al., 2012, Proceedings of the EFTF, Gothenburg, Sweden, arXiv:1206.6239.
\end{abstract}

Keywords. gravitation, time, methods: data analysis

\section{The ACES-PHARAO mission}

The Atomic Clocks Ensemble in Space - ACES-PHARAO mission (C. Salomon et al., 2007, IJMPD, 16, 2511) - will be installed on board the International Space Station (ISS) in 2015 and will realize in space a time scale of very high stability and accuracy. This time scale will be compared to a ground clock network thanks to a dedicated two-way microwave link. The ACES mission will demonstrate the capability to perform phase/frequency comparison between space and ground clocks with a resolution at the level of 0.4 ps over one ISS pass (300 s), 8 ps after 1 day, and 25 ps after 10 days of integration time (see Fig. 1). For that purpose our team is developing advanced time and frequency transfer algorithms.

The altitude difference between the ACES-PHARAO clock and ground clocks will allow to measure the gravitational redshift with an unprecedented level of accuracy, as well as looking for a violation of Lorentz local invariance. Several ground clocks based on different atomic transitions will be compared to look for a drift of the fine structure constant. Moreover, the mission will pave the way to a new type of geodetic measurement: the gravitational redshift will be used to measure gravitational potential differences between distant clocks, with an accuracy of about $10 \mathrm{~cm}$.

\section{The micro-wave link}

The micro-wave link will be used for space-ground time and frequency transfer. It is composed of three signals of different frequencies: one uplink at frequency $f_{1} \simeq 13.5 \mathrm{GHz}$, and two downlinks at $f_{2} \simeq 14.7 \mathrm{GHz}$ and $f_{3}=2.2 \mathrm{GHz}$. Measurements are done on the carrier itself and on a code which modulates the carrier. The link is asynchronous: a configuration can be chosen by interpolating observables. The so-called $\Lambda$-configuration minimizes the impact of the space clock orbit error on the determination of the desynchronisation (Duchayne et al., 2009, A\&A, 504, 653). We sketch on Fig. 2 the space-time diagram of the two signal $f_{1}$ and $f_{2}$ in a $\Lambda$-configuration. We define the SYRTE Team

$\dagger$ E-mail: Pacome.Delva@obspm.fr 


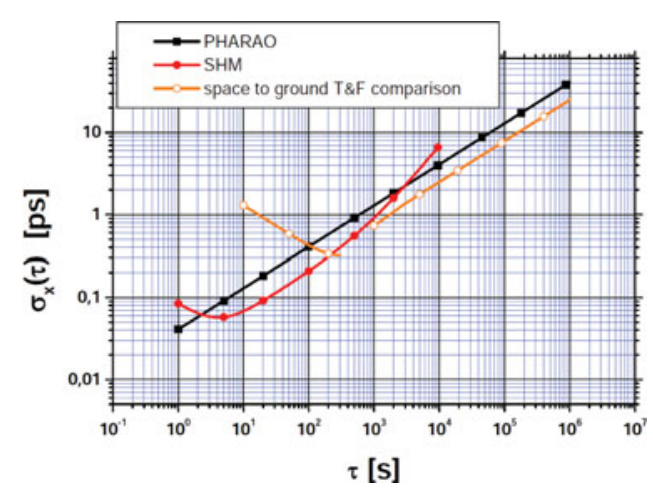

Figure 1. Performance objective of the ACES clocks and the ACES space-ground time and frequency transfer expressed in time deviation

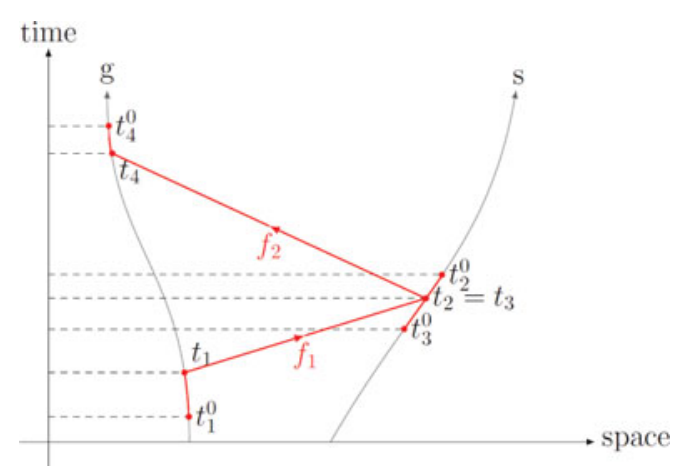

Figure 2. Signal $f_{1}$ is generated at coordinate time $t_{1}^{0}$, is emitted by the ground antenna at time $t_{1}$, received by the space antenna at $t_{2}$ and arrives at the receiver modem at $t_{2}^{0}$. For signal $f_{2}$ the sequence is $\left(t_{3}^{0}, t_{3}, t_{4}, t_{4}^{0}, t_{8}^{0}\right)$. In the $\Lambda$-configuration interpolation is such that $t_{2}=t_{3}$.

(ST) observables by $\Delta \tau\left(\tau_{e}\right)=\tau_{e}-\tau_{r}$, where $\tau_{e}$ is the proper time of emission of the signal and $\tau_{r}$ the proper time of reception. It can be linked to desynchronisation:

$$
\tau^{s}\left(t_{2}\right)-\tau^{g}\left(t_{2}\right)=\frac{1}{2}\left(\Delta \tau_{\mathrm{mo}}^{g}\left(\tau^{g}\left(t_{4}^{0}\right)\right)-\Delta \tau_{\mathrm{mo}}^{s}\left(\tau^{s}\left(t_{2}^{0}\right)\right)+\left[T_{34}-T_{12}\right]^{g}\right)
$$

where $t$ is coordinate time, $\Delta \tau_{\text {mo }}$ are the ST observables corrected for the delays in the cable between the clock and the antenna at transmission and at reception, $T_{i j}=t_{j}-t_{i}$ and $[.]^{g}$ is the coordinate time to the ground clock proper time transformation.

The time-of-flights $T_{34}$ and $T_{12}$ can be calculated from the known orbits of the clocks, accounting for the tropospheric, ionospheric and Shapiro delays. The observables from the two downlinks can be used to determine the Total Electronic Content (TEC) of the atmosphere along the line-of-sight, in order to correct for the ionospheric delay. The twoway configuration cancels the tropospheric delay, which does not depend on the signal frequency at this level of accuracy.

The basic observables of the modem developed by TimeTech (TT observables) are different from the ST observables. A PPS signal (one Pulse Per Second), a 12.5 PPS (one pulse every $80 \mathrm{~ms}$, the period of measurements), and a periodic signal (either code at $100 \mathrm{MHz}$ or carrier) are generated in the emitter and the receiver. When received, the periodic signal is mixed with a local oscillator signal not far from the received frequency, and filtered to obtain the low frequency part of the beatnote. The beatnote frequency is around $195 \mathrm{kHz}$ for code and $729 \mathrm{kHz}$ for carrier. The receiver modem records the time of the first ascending zero-phase of the beatnote signal after the 12.5 PPS signal, and it counts the number of ascending zero-phase during one $80 \mathrm{~ms}$ sequence. The link between the TT and ST observables is detailed in Delva et al., 2012, Proceedings of the EFTF, Gothenburg, Sweden, arXiv:1206.6239.

\section{Conclusion}

The ACES simulation of the micro-wave link is written in its first version, and used to assess our pre-processing software. The design of the data analysis software has been done in a modular way, and most of the building blocks are ready. Several questions remain, e.g. how to solve the phase ambiguity for the carrier observable. 